\title{
SOBRE EL CONCEPTO DE VARIACIÓN FRASEOLÓGICA
}

\author{
M. Belén Alvarado Ortega \\ Universidad de Alicante. Grupo GRIALE \\ Belen.Alvarado@ua.es
}

\begin{abstract}
Resumen
En este trabajo presentamos un estudio de uno de los fenómenos fraseológicos más difíciles de delimitar, la variación. Así, pese a que muchos autores han intentado delimitar el término, no existe congruencia entre ellos. De ahí que intentemos analizar este tipo de propiedad que pueden presentar las UFs.

PALABRAS CLAVE: variación, unidades fraseológicas, fórmulas rutinarias.
\end{abstract}

\begin{abstract}
This article presents an analysis of one of the most controversial phraseological phenomenon: variation. Therefore, although some authors have attempted to define this term, there is no consistency among them. Consequently, we will analyze this type of property shown by some phraseological units.

KEYWORDS: variation, phraseological unit, conversational routines.
\end{abstract}

\section{Introducción}

En este trabajo proponemos definir y delimitar una de las propiedades que pueden presentar las unidades fraseológicas (UFs): la variación. La complejidad que presenta esta propiedad no fundamental ${ }^{1}$ se debe a que, a pesar de las numerosas reflexiones que hay en la bibliografía, no se ha llegado a unos rasgos comunes que puedan definir a una UFs como variante de otra. Por ello, resulta complicado discernir si una UFs presenta variantes en su realización o, por el contrario, son UFs diferentes. Así pues, proponemos en este artículo deslindar esta característica que pueden manifestar las UFs y, en concreto, las fórmulas rutinarias (;madre mía!, ;vaya tela!, etc.). Tomamos como ejemplos contextos extraídos del CREA y del Corpus de Val.Es.Co.

Con el fin de evitar errores terminológicos y conceptuales, definimos $U F$ como la combinación formal y psico-lingüísticamente estable de unidades léxicas compuestas por dos o más palabras que forman parte de la competencia léxica de los hablantes, cuyo límite superior se encuentra en el nivel de la oración compuesta y que, a su vez, puede poseer idiomaticidad (Alvarado 2008: 30). Mientras que sobre el concepto de fórmula rutinaria asumimos con Corpas (1996) que se encuentran inmersas dentro de un grupo más amplio de UFs denominado enunciados fraseológicos ${ }^{2}$ en los que se incluyen tanto a las paremias,

1 Consideramos propiedades fundamentales de las UFs la idiomaticidad (la suma del significado de sus componentes no es igual a la suma total de la unidad) y la fijación (presenta estabilidad en su formación y en su uso) (Alvarado 2008).

2 Corpas (1996) diferencia dentro de este tipo de UFs, los enunciados fraseológicos, dos subgrupos, las paremias y las fórmulas rutinarias. 
compuestas por refranes, enunciados de valor específico y citas, como a las fórmulas rutinarias. A nuestro modo de ver, las fórmulas rutinarias son UFs compuestas por dos o más palabras que se encuentran, en cierto modo, ritualizadas, y cuyo límite superior se encuentra en la oración compuesta. Este hecho ocasiona que posean fijación formal y psico-lingüísti$\mathrm{ca}^{3}$, potencial idiomaticidad e independencia en algún grado, como enunciados fraseológicos que son.

A continuación, nos centramos en el fenómeno de la variación.

\section{Breve repaso bibliográfico sobre la variación}

En general, han sido muchos los estudios que se han ocupado del concepto de variación fraseológica, entendido como la alterabilidad de la UFs en su formación; sin embargo, cada investigador ofrece una serie de rasgos diferentes para definirla, sin existir acuerdo entre ellos, como veremos posteriormente. En principio, esta propiedad no es un rasgo fundamental de deben presentar las UFs, como puede ocurrir con la fijación, la idiomaticidad o la independencia ${ }^{4}$, pero es una propiedad que pueden adquirir en su uso cotidiano. Diversos autores (Casares o Zuluaga, entre otros) han intentado explicar qué entienden por variación. Por eso retomamos algunas manifestaciones, ordenadas de forma cronológica, sobre este concepto para llegar a una definición propia de qué es la variación y cómo se manifiesta en las UFs.

Casares ([1950] 1969), como lexicógrafo que es, no se refiere directamente a este término en cuanto a fenómeno fraseológico; sin embargo, en algunos momentos de su obra interpretamos que alude a esta propiedad:

Se armó la de San Quintín. Esta expresión sería igualmente un ejemplo de frase proverbial si pudiéramos estar ciertos de que el uso no admite otras formas del verbo armar. $\mathrm{Si}$, por el contrario, fuese normal decir se ha armado, o se armará, o se está armando la de San Quintín, nos hallaríamos ante una locución de las que hemos clasificado como verbales: armar la de San Quintin. (Casares [1950]1969: 189).

En la explicación que muestra Casares para discernir entre frase proverbial y locución encontramos que una de las características que se debe tener en cuenta es si admite o no cambios en su estructura interna. Así, si admite cambios, según Casares, nos encontraríamos ante una locución verbal, mientras que si no los admite estaríamos ante una frase proverbial. Por tanto, para este autor las frases proverbiales no admitirían ningún tipo de variación interna, ya que es lo que las diferencia de las locuciones.

3 Consideramos que esta clase de fijación se asocia con la idea de institucionalización o convencionalización que enuncia Corpas (1996: 21), es decir, se trata de la estabilidad en su reproducción y en la frecuencia de uso que presentan las UFs en general y las fórmulas rutinarias en particular. Los hablantes las recuerdan y las producen en bloque como una única unidad léxica. La fijación formal sería la propiedad prototípica y fundamental junto a la fijación psico-lingüística, mientras que la fijación semántico-pragmática seria una propiedad menos prototípica y más periférica para las fórmulas rutinarias en general, ya que su aparición viene condicionada por el tipo de fórmulas rutinarias que se analicen (Alvarado 2008: 109).

4 Véase Alvarado (2008: 140). 
Con respecto al carácter inalterable de los modismos, Casares ([1950] 1969: 211) afirma:

Se ha indicado que los modismos son siempre locuciones compuestas que tienen el carácter de "clichés", donde no es posible alterar ni los componentes, ni la relación sintáctica entre unos y otros. Esto es cierto, efectivamente en muchos casos, pero no es aplicable a todos. La forma antigua de levantar el codo no era ésta, sino la de alzar el codo.

En estas palabras, el autor hace referencia a la idea de variación para los modismos y clichés, y afirma que en muchos casos son inalterables, pero en otros sí que admiten algún tipo de cambio interno. No debemos olvidar que Casares era lexicógrafo, por tanto, sus aproximaciones al término todavía tienen mayor relevancia si cabe, ya que observa que existe el fenómeno de la variación, que afecta a la fraseología, desde su perspectiva lexicográfica.

Una de las primeras aportaciones fraseológicas a la variación en español la ofrece el fraseólogo Zuluaga en 1980. Este autor (Zuluaga 1980: 109) dedica un apartado de su obra a distinguir entre "auténticas" variantes (o variantes en sentido estricto) y variaciones (o variantes en sentido amplio). Sobre las variantes en sentido estricto aporta los siguientes rasgos:

- Deben darse dentro de una misma lengua funcional, por ejemplo, tomar las de Villadiego, coger las de Villadiego son iguales en España, pero no en Argentina.

- No pueden presentar diferencias de sentido.

- Son libres, es decir, son independientes de los contextos, aunque no encontramos variantes del tipo Ignacio y Antonio, Antonio e Ignacio.

- Son parcialmente idénticas en su estructura y en sus componentes, la variante se produce por sustitución de una parte de la expresión. La sustitución misma es fija, ya que los elementos sustituyente y sustituido están fijados previamente. Así, tomar las de Villadiego, coger las de Villadiego son variantes porque sólo cambia una unidad.

El autor sitúa al margen de las variantes en sentido estricto los esquemas fraseológicos ${ }^{5}$ o moldes sintácticos rígidos, que apenas poseen libertad combinatoria y que se explican mediante el concepto de fijación.

Entre las variantes en sentido amplio se encontrarían:

- Las transformaciones reales o modificaciones de unidades fraseológicas (UFs) que llevan consigo cambio de significado. Así, ponerse al corriente y estar al corriente son UFs diferentes porque tienen distinto significado. La primera significa informarse, y la segunda conocer, según Zuluaga (1980: 107).

- Las UFs cuya estructura o cuyos componentes sean, en su totalidad, diferentes, y signifiquen lo mismo serán sinónimas, como tomar las de Villadiego y poner pies en polvorosa.

5 Zuluaga (1980: 191) define los enunciados fraseológicos como expresiones fijas equivalentes o superiores a la frase cuyo "rasgo definitorio es el funcionar como unidades comunicativas mínimas con sentido propio, enunciadas por un hablante entre dos pausas y en unidades de entonación distintas". 
- Las variaciones regionales o diatópicas no son consideradas variantes en sentido estricto, como hacer novillos que se da en España frente a hacer vaca que aparece en Perú. Ambas con el significado de faltar a la escuela.

- Las variaciones socioculturales, como me importa un chorizo que se da en Venezuela o Colombia equivalente a me importa un bledo que aparece en España, no son variantes en sentido estricto.

- Las variaciones diafásicas tampoco son variantes en sentido estricto, puesto que se dan en diferentes modalidades de la lengua, como sentidísima condolencia y sentido pésame.

- Las UFs con casillas vacías tampoco se consideran variantes en sentido estricto, como a... anchas o a ....azo limpio.

Zuluaga (1980: 107) afirma, además, que los miembros de las llamadas series (de buena $f e$ - de mala fe) se deben considerar como expresiones diferentes, ya que su significado es diferente y, a veces, opuesto. Por tanto, desde la perspectiva funcionalista, encontramos en este autor la primera distinción entre diferentes tipos de variantes: variante (o variante en sentido estricto), variación (o variante en sentido amplio) y esquema fraseológico. Sobre estos tipos se centrarán, como veremos, posteriores estudiosos del tema.

Hernando (1990: 537) dedica un breve apartado de su trabajo para hablar de este fenómeno. Retoma las ideas de Zuluaga y habla de variantes stricto sensu consideradas dentro de una misma lengua funcional "son parciales (no se sustituye toda la expresión, sino parte de ella o alguno de sus componentes) y no presentan diferencias de sentido". El autor (Hernando 1990: 537) afirma que:

La sustitución misma es fija, efectuándose, según los casos: mediante un solo elemento, con exclusión de los demás del mismo campo léxico: todo queda en casa (familia); con los diversos elementos del mismo campo: tomar (coger, asir, agarrar) el cielo con las manos; con elementos de campos diferentes, pero pertenecientes a la misma clase léxica: se las da (echa) de...; con elementos que sólo tienen en común el pertenecer a la misma categoría semántica: aqui fue (ardió) Troya; con una negación respecto a una afirmación: (no) me importa un pepino, o un plural en relación con un singular: llevarse de calle(s), sin valor opositivo.

Como vemos, Hernando ha retomado algunas ideas de Zuluaga y las ha modificado, ya que trata como variantes en sentido estricto aquellas que Zuluaga había considerado en sentido amplio, como hemos visto en párrafos anteriores.

Corpas (1996: 27) trata el fenómeno de la variación partiendo de los presupuestos de Zuluaga y distingue entre variantes y modificaciones. La autora define las variantes según las características que Zuluaga ofrecía en sentido estricto (pertenecer a una misma lengua funcional, no presentar diferencias de significado, etc). Además, distingue las variantes de las simples variaciones por derivación o transformación. Tampoco considera variantes las UFs que forman parte de series con significado opuesto generalmente, las variaciones diatópicas, las variaciones diafásicas ni las UFs con casillas vacías. Por tanto, todas ellas quedan excluidas de su propuesta. Así, según la autora, todo queda en casa y todo queda en familia serían variantes, pero no todo queda en el hogar. 
Las variantes se deben distinguir de las modificaciones (Corpas 1996: 29), puesto que las primeras carecen de la intencionalidad del hablante, mientas que las segundas tienen que ver con la manipulación creativa e intencionada del mismo. Las modificaciones se pueden interpretar mejor por parte del oyente cuando la UFs está completamente fijada en la lengua. De esta manera, es más fácil que su efecto sea reconocido por el interlocutor, como vemos en la siguiente estructura modificada, a la última va la vencida cuando el verdadero esquema es a la tercera va la vencida.

Por tanto, en Corpas encontramos reflejadas las ideas de Zuluaga en cuanto al término de variación y sus características, con una diferencia terminológica, ya que la autora prefiere denominar variantes a las variantes en sentido estricto de Zuluaga y modificaciones a las variantes en sentido amplio del mismo autor. Esta distinción terminológica será la que tomemos en nuestra definición, como veremos más adelante.

García-Page (1996: 479) define variante como una construcción prefabricada que admite la sustitución léxica de alguno de sus componentes sin alterar su significado. En su definición aparece la idea de fijación de significado y de variabilidad léxica restringida, que más tarde desarrollaran otros autores como Koike (2001). Distingue las variaciones de las variantes, al igual que Zuluaga (1980), entre las que se encuentran:

- Las posibles alternativas léxicas (sinónimos, etc.).

- Las expresiones fijas sinónimas.

- Las fórmulas de relleno.

- Los antónimos.

— Las variaciones espontáneas e individuales.

Años más tarde, García-Page (1999: 225) afirma que "las UFs se caracterizan por su fijación, por su petrificación en una forma sólida, inmodificable (...), pero un número no precisamente escaso de fraseologismos toleran variaciones de naturaleza diversa"; entre los ejemplos que presenta se encuentran importar un pepino, importar un rábano, importar un bledo, etc. que serían variantes de una misma UFs. Además, recoge la clasificación que otros autores han dado para clasificar los distintos tipos de variantes que pueden darse (variantes léxicas, morfológicas, gramaticales y gráficas) y se centra en los cambios morfológicos que pueden tener las UFs y son de dos tipos: flexivos o derivativos. Para el autor las abreviaturas fraseológicas se encontrarían dentro de las variantes gramaticales, ya que afectan a la categoría de la palabra.

Zamora (1998: 110) afirma que desde el punto de vista morfológico y sintáctico algunas UFs, como las frases idiomáticas pragmáticas, presentan restricciones:

En líneas generales se puede afirmar que admiten en casos muy aislados variables categoriales de sus componentes y de conectores, pero en su mayoría son invariables al poseer una capacidad de conmutación muy limitada, al presentar fijación en el orden de sus constituyentes y no permitir generalmente ninguna transformación estructural, ni ninguna inserción, sustitución o supresión (Zamora 1998: 110).

El autor demuestra, teniendo en cuenta los diferentes tipos de variables que considera (variables lexicales, morfológicas y sintácticas), que las frases idiomáticas pragmáticas son en su mayoría invariables y no admiten ningún tipo de inserción ni supresión. 
Otro de los autores que trata este fenómeno es Moon (1998: 120), que analiza de forma extensa este concepto en la lengua inglesa y enumera diferentes tipos de variación: variación léxica (variaciones de verbos, nombres, adjetivos, conjunciones, de registros, etc.), variación sistemática, variación y esquemas, relaciones de antonimia y sinonimia, realizaciones libres, esquemas idiomáticos e interrupciones e inserciones. Para esta autora, la variación sería un concepto mucho más amplio que el que hemos visto en otros investigadores, ya que incluye en ella no sólo las variaciones léxicas, sintácticas y morfológicas, sino también las variaciones de registro y las relaciones semánticas de antonimia y sinonimia que otros autores descartaban.

Por su parte, Burger (2000: 105) afirma que la variedad "puede entenderse en relación a la fraseología como una variabilidad sociocomunicativa". Este autor elabora una clasificación de los diferentes tipos de variedad segủn aspectos diacrónicos, diatópicos, diastráticos y diafásicos, y la relación que mantienen con los diccionarios, ya que las "variantes codificadas, y por ello toleradas como pertenecientes a la lengua estándar, no se corresponden con la diversidad de variantes que aparecen en la comunicación oral". Diferencia entre variedad y modificación, según la variación sea intencionada, como imanda cojones! por imanda narices! (modificación), o no, como confundir el tocino con la velocidad / confundir la velocidad con el tocino (variedad), al igual que hacía Corpas (1996).

Por tanto, este autor realiza una clasificación social de las variedades que se pueden dar en las UFs, las cuales se corresponden con las variaciones en sentido amplio (no consideradas variantes) de Zuluaga (1980: 106).

Por otra parte, Koike (2001: 77) define variantes fraseológicas como:

Unidades fraseológicas que, manteniendo el mismo significado, son parcialmente idénticas en su estructura y en sus componentes. Son variantes, pues, hacer la pelota, hacer la rosca, hacer la barba y hacer la pata, pero chupar las medias no es variante de hacer la pelota, sino una locución sinónima.

Este autor realiza un estudio de la variación fraseológica en el español, tanto de España como del mundo hispánico, dentro del proyecto Varilex ${ }^{6}$ en el que se llevó a cabo la mayor encuesta de variación léxica del mundo. En su trabajo, analiza la distribución geográfica de las diferentes locuciones recogidas en la encuesta, los tipos de variaciones, la homonimia y la productividad fraseológica. Afirma Koike (2001: 88) que:

Las variantes fraseológicas admiten diversas clases de alternativas dentro de la misma estructura sintáctica sin presentar diferencias de significado. Las alternativas pueden ser morfológicas, morfosintácticas, sintácticas y léxicas.

De esta manera, presenta diferentes tipos de variación. Entre los casos de variación morfológica destaca, entre otros, la variación por derivación y en el número gramatical. En la variación sintáctica destaca la que concierne al uso de las preposiciones, artículos, orden de los componentes y empleo de formas acortadas, entre otras. Sobre la variación

6 Véase http://lingua.cc.sophia.ac.jp/varilex/index.php. 
léxica afirma que consiste principalmente en la sustitución de uno de los componentes de la UFs. Aunque su propuesta la enfoca directamente a las locuciones, veremos que también es válida para las UFs en general, ya que los diferentes tipos de variación se pueden aplicar a todas ellas.

Autores como Ortega y González Rey (2005: 92) definen variación fraseológica como:

El fenómeno que se produce cuando una UF, a pesar de estar fijada, se materializa en "formas" distintas sin que dicho cambio conlleve alteraciones en el significado fraseológico, teniendo en cuenta que para ser llamada variante debe presentar más coincidencias que diferencias.

Estos autores resumen en tres puntos el modo en el que se ha entendido la variación fraseológica hasta el momento (Ortega y González Rey 2005: 97):

- Como relación que existe entre UFs sinonímicas, aunque Zuluaga (1980) las trata como UFs distintas.

- Como relación que existe entre UFs sinonímicas que comparten una estructura semejante. De esta manera se concibe en el proyecto Varilex.

- Como relación que existe entre las variantes en sentido estricto, tal y como lo entiende Zuluaga (1980).

A todo ello hay que añadir que proponen una clasificación que se basa en Carneado (1983), retomada más tarde por Koike (2001), y distinguen entre modificaciones morfológicas, sintácticas, léxicas y por extensión. Para ellos, las modificaciones morfológicas son aquellas que producen cambios en las subcategorías de las UFs; las modificaciones sintácticas son las variaciones en las que hay trueque entre dos elementos, cambia la estructura sintáctica, etc.; las modificaciones léxicas tienen que ver con el cambio de significado e incluyen las series de Zuluaga (1980: 107), que consideraba como expresiones diferentes, sin embargo, los autores las consideran variantes por el modo en el que se aprenden; y las modificaciones por extensión se dan en los casos en los que la UF tiene una versión más extensa y otra más abreviada.

Además, Ortega y González Rey (2005: 103) retoman el término de esquemas fraseológicos de Zuluaga (1980), y añaden que lo utilizan para

designar aquellas combinaciones de palabras fijadas a las que se les añade un sintagma u oración (...). Estos esquemas cumplen con dos requisitos importantes para ser analizados como variantes: la equivalencia y la semejanza formal parcial.

Por tanto, en este sentido difieren de Zuluaga, puesto que este autor consideraba que los esquemas fraseológicos se situaban al margen de las variantes en sentido estricto ya que eran UFs fijas, mientras que Ortega y González Rey los tratan como variantes.

Montoro del Arco (2006: 58) retoma el problema terminológico entre variación y variante. Para este autor las variantes se basan en la sustitución paradigmática, mientras que las variaciones dependen de la inserción de la UFs en la cadena sintagmática. Así, considera que tanto las series fraseológicas como las variaciones diatópicas, diastráticas y diafásicas son variantes de un mismo fraseologismo. 
En la siguiente tabla resumimos las propuestas de los investigadores que hemos analizado en este epígrafe:

\begin{tabular}{|l|l|}
\hline \multirow{2}{*}{ Zuluaga (1980) } & Variantes en sentido estricto (tomar las de Villadiego/coger las de Villadiego) \\
\cline { 2 - 3 } & Variantes en sentido amplio (me importa un chorizo/ me importa un bledo) \\
\hline Hernando (1990) & Variantes en sentido estricto (tomar/asir/coger el cielo con las manos) \\
\hline \multirow{2}{*}{ Corpas (1996) } & Variantes (todo queda en casa/todo queda en familia) \\
\cline { 2 - 3 } & Modificaciones (a la última va la vencidal a la tercera va la vencida) \\
\hline García-Page (1996) & Variantes (importar un pepino/ importar un rábano) \\
\hline Zamora (1998) & Variables (léxicales, morfológicas, sintácticas) \\
\hline Moon (1998) & Variación (léxica, sintáctica, sistemática, etc.) \\
\hline \multirow{2}{*}{ Burger (2000) } & $\begin{array}{l}\text { Variedad (confundir el tocino con la velocidad/ confundir la velocidad con } \\
\text { el tocino) }\end{array}$ \\
\cline { 2 - 3 } & Modificación (jmanda narices!/imanda cojones!) \\
\hline Koike (2001) & Variantes (morfológica, sintáctica, etc.) \\
\hline \multirow{2}{*}{$\begin{array}{l}\text { Ortega y González } \\
\text { Rey (2001) }\end{array}$} & Variantes (en sentido estricto y esquemas fraseológicos) \\
\cline { 2 - 2 } & Modificaciones (léxicas, sintácticas, etc.) \\
\hline \multirow{2}{*}{$\begin{array}{l}\text { Montoro del Arco } \\
\text { (2006) }\end{array}$} & Variantes (sustitución paradigmática) \\
\cline { 2 - 2 } & Variación (cadena sintagmática) \\
\hline
\end{tabular}

Tabla 1. Resumen de propuestas.

En la tabla 1 observamos que en todos los autores analizados, los términos y los conceptos relativos al fenómeno de la variación cambian constantemente, ya que, aunque denominan bajo el mismo nombre al fenómeno general, cada uno de ellos aporta rasgos diferentes. De ahí que resulte complicado discernir si una misma UFs presenta variantes o son UFs distintas.

Con el fin de facilitar el trabajo de posteriores investigadores en el campo de la fraseologia, y teniendo en cuenta las investigaciones previas, creemos conveniente realizar una distinción terminológica y conceptual, basada en reflexiones propias, relativa al fenómeno de la variación.

\section{La variación: variantes y modificaciones}

En nuestra opinión (Alvarado 2008), consideramos primeramente la distinción terminológica que ha propuesto Corpas (1996) entre variante y modificación, puesto que permite distinguir si estamos o no ante UFs diferentes ${ }^{7} \mathrm{y}$ creemos innecesario crear nuevos términos.

7 En el trabajo realizado en 2008, analizamos un corpus de 1400 ocurrencias de 90 fórmulas rutinarias diferentes, extraídas del Corpus de Val.Es.Co., del COVJA y del CREA. De todas ellas, observamos que algunas eran variantes de otras, como veremos en los ejemplos. 
Sin embargo, la definición de dichos términos varía, ya que consideramos que la variación es el término amplio que engloba tanto a las variantes como a las modificaciones.

Las variantes son ajenas al efecto que la UF tenga en sí misma, es decir, no presentan una intencionalidad extra por parte del hablante para provocar determinados efectos en su oyente. Por el contrario, las modificaciones sí que gozan de esa manipulación e intencionalidad por parte del hablante y, de esta manera, se consigue que la UF tenga un significado ajeno al habitual. Por tanto, estos casos no se consideran variantes. Definimos en los puntos siguientes estos fenómenos, modificaciones y variantes, como partes de la variación en las UFs.

\subsection{Modificaciones}

Consideramos que el fenómeno de la modificación está estrechamente ligado con la idea de desautomatización, entendida como la manipulación en la forma o contenido de una UF para producir determinados efectos (Ruiz Gurillo 1997: 21). Para que el hablante pueda producir una modificación cuando utiliza la UF en su enunciado, ésta debe tener un alto grado de fijación formal para que el oyente la identifique sin dificultades, como observamos en el siguiente ejemplo en el que una mujer llama a un programa de radio para contar lo que le hizo su suegra al volver de un viaje que hizo a Portugal:

(1) $\mathrm{A}^{8}$ : Chica, y me dijo que no me preocupara que me había traído una manta de Portugal, me lo prometió antes de irse. Se puso a buscar y se dio cuenta que se la había regalado a Asun, una vecina.

B: Un descuido que tuvo la mujer, pero claro lo prometido es deuda.

A: ¿Lo prometido es deuda? Mejor lo prometido es duda.

B: No creo que no te la quisiera dar.

A: No conoces a mi suegra...

(CREA, "A vivir que son dos días", 02/11/96, Cadena SER)

En el ejemplo anterior observamos cómo la UF, lo prometido es deuda, ha sido modificada por el hablante para mostrar que la actitud de su suegra no fue fortuita, ya que A piensa que lo hizo a propósito. Si observamos el ejemplo, A toma como punto de partida en su modificación la intervención anterior en la que $\mathrm{B}$ reproduce la UF real lo prometido es deuda para mostrarle que no está de acuerdo con lo que ha hecho su suegra. A retoma las palabras de B y corrige la UF para crear una modificación, que explica mejor la actitud de la suegra.

En (2) dos amigos están hablando de un tercero que se ha llevado unos libros sin permiso:

(2) A: Se ha llevado mis libros por el morro cuando yo no estaba.

B: Eso es lo que se llama somorra y gomorra.

A: jajajaja

(http://foros.atomix.vg/showthread.php?p=2504201)

8 El CREA no presenta distinción entre participantes e intervenciones, por eso hemos reconstruido la conversación como pensamos que fue. 
En este ejemplo B emplea una UF de forma desautomatizada y modificada, ya que le sirve para hacer un juego de palabras con morro (el que le ha echado el amigo para quitarle los libros) y gorra (conseguir algo gratis). Por tanto, la UF original sodoma y gomorra se ha modificado para expresar el malestar de B. Además, observamos que A ha captado perfectamente esa modificación, como se aprecia en la última intervención.

Cuando se manipula una UF, el coste de procesamiento es mayor para el oyente, por tanto hablante y oyente deben tener un conocimiento compartido de la realidad que les rodea para que se garantice la perfecta comunicación. Además, el fenómeno de modificación o desautomatización puede ser el aliciente para producir, en ocasiones, otros efectos en el discurso como puede ser la ironía verbal (Alvarado 2007).

\subsection{Variantes}

Consideramos que las variantes se dan en las UFs cuando presenta un solo cambio léxico en su estructura, bien sea por adición o reducción, bien por sustitución, sin alterar su componente principal, su significado, en los contextos en los que puede aparecer. Además, incluiríamos dentro de las variantes los cambios de registro y los cambios de código, siempre y cuando sean entendidos por el oyente como tales.

Por cambios léxicos entendemos la sustitución o la adición de alguno de sus componentes, sin que influya en el elemento que posee la carga ilocucionaria. Para ilustrar las variantes por sustitución de algún componente observamos, por ejemplo, el caso de $y$ eso, y todo eso. Aunque algunos autores las considerarian UFs diferentes (Zuluaga 1980), para nosotros se trata de la misma fórmula con diferentes alteraciones, ya que su valor en la conversación es el mismo en todos los contextos en los que se produce. Como observamos en (3) en el que varios interlocutores hablan sobre una persona ausente en la conversación:

(3) $205<$ E2 $>$ : O sea, </simultáneo $>$ cuarenta, no sé si han sido cuarenta, y me estoy yendo por lo alto y $(\rightarrow>)$... no, sí, me estoy yendo por lo alto. Y $(\rightarrow>)$... y el caso es que ella decía que <estilo indirecto> el novio estaba trabajando... y que (-->)... vamos, para estar en casa, pues que ella quería ganar dinero y $(->)$... si se quería comprar ropa ($\rightarrow$ ) $<$ estilo indirecto $>$... pos $<$ sic $>$ por lo que lo hacemos to $<$ (d) $>$ o el mundo, ino? Y nada, y eso ¿estar ocho horas trabajando... cuarenta mil pesetas?

(CREA, COVJA, Grupo 1, pág. 64)

En (3) E2 enuncia la UFs y eso para concluir su intervención. Así, observamos que esta fórmula se utiliza se utiliza para reconducir la información hacia una conclusión final, ya que como vemos va precedida además de y náda, que es otra fórmula discursiva. Con este valor conclusivo podría haber aparecido otra variante, como y todo eso, ya que no se alteraría su significado ni su función en la conversación.

Los cambios de registro, considerados variantes, que se producen en la lengua afectan, por ejemplo, a la fórmula ime cachis en la mar!, que sería menos vulgar dentro del registro coloquial que ime cago en la mar!, a pesar de que estamos ante una misma fórmula. Como vemos en el ejemplo (4) en el que varias amigas hablan sobre sus hijos adolescentes: 
(4) C: con mis hijos me pasó/ estaban sentaos ahí en el- en el banco// viene la novia de mi hijo el mayor y le dice a mi madreee ¿me da usté los papeles de Fran de la moto? que es quel see le ha pinchao y la policia como no lleva los papeles/ quieren los papeles $\uparrow$ que se creen que es robadal/ al momento vienen los amigos de mi Javi// mire $\downarrow$ que me dé los papeles dee la moto de Javi§ A:

C:

$\S$ que $\rightarrow$ ¡me cagüen la mar! yo digo esto es que se m' ha matao un HIJO MIO y estos no me [lo quieren DECIR =]

D: $\quad$ [y ya está $\quad$ sí]

(Briz y grupo Val.Es.Co., 2002: 239 [H.25.A.1: 268-277])

En (4) C enuncia la fórmula jme cagüen la mar! para mostrar enfado ante la situación que está reproduciendo, ya que pensaba que le había sucedido algo a su hijo. Además, observamos que esta fórmula es la reducción de una expresión más larga (ime cago en la mar salada!) y que posee variantes como ime cago en la puta!, ime cago en la hostia!, ime cago en la leche!, ime cachis la mar!, etc. Todas ellas tienen el mismo significado en la conversación, expresar enfado, sólo se ha alterado el registro y se ha producido un cambio léxico en cada una de ellas; por tanto, son variantes.

Los cambios de código que se pueden producir en la lengua funcional también los consideramos variantes ${ }^{9}$. Así, si estamos ante un grupo de hablantes binlingües (castellano/valenciano) y en la conversación aparece imare meua!, los oyentes entienden que la fórmula es un cambio de código y que se corresponde con imadre mía!, que aparece en la conversación para mostrar sorpresa. Por tanto, en este caso no estaríamos ante UFs distintas, sino ante variantes de una misma UF, ya que el oyente interpreta correctamente su significado. Como vemos en (5), donde una madre habla con su hija sobre un viaje que va a hacer próximamente:

(5) A: [IMAGINO] QUE a las seis o las siete nos iremos

B: a las seis es de noche ¿eh?///(4") ¡ay/ mare meua!// ya no estaré yo tranquila// os podíais ir a una casa aquí en Castellón más $\rightarrow$ cerquita

A:

$\S$ ¿DÓONDE?

(Briz y grupo Val.Es.Co., 2002: 328[VC.117.A.1:254-258])

En (5) el hablante, que es bilingüe, enuncia la fórmula jmare meua! para mostrar indignación ante lo que le está diciendo su hija, A, que queda intensificado con el uso de la interjección jay! De este modo, A entiende la fórmula imare meua! correctamente y sabe que la ha expresado para mostrar sorpresa e indignación, que es el mismo significado que tiene ;madre mía! Por tanto, en este caso no estamos ante fórmulas distintas, sino ante variantes de una misma UF.

En estos ejemplos observamos que no hay intencionalidad por parte del hablante de añadir un nuevo matiz a los significados de las UFs, sino que simplemente muestra el estado de ánimo del mismo. Sin embargo, en las modificaciones hemos observado que el hablante añade al significado de las UFs un nuevo matiz de forma intencionada, lo que manifiesta que se trata de un hecho diferente dentro de la variación.

9 Este tipo de variante es marginal, pero aparece en zonas bilingües, como hemos apreciado en el COrJA y en el Corpus de Val.Es.Co. 


\section{Conclusiones}

En este trabajo hemos intentado delimitar el fenómeno de la variación fraseológica y las diferencias conceptuales que existen entre variantes y modificaciones, ya que, aunque no son propiedades fundamentales de las UFs, sí que pueden aparecer en ellas. Por eso, a modo de conclusión, esbozamos las siguientes ideas:

- La variación es el fenómeno general que engloba a las modificaciones y a las variantes.

- Las modificaciones son un fenómeno intencional por parte del hablante y pueden producir diferentes efectos en el oyente. Para que esto se produzca, la UFs tiene que estar fijada.

- Las variantes son UFs con idéntica estructura, significado y con un solo cambio léxico en ellas ( $y$ eso, $y$ todo eso).

- Las UFs sinónimas no tienen por qué ser variantes, por ejemplo imadre mia! y iqué me dices! muestran sorpresa y tienen la misma función en la conversación, es decir, son sinónimas, pero no son variantes.

- Toda UF variante es sinónima, por ejemplo ime cachis en la mar! y jme cago en la mar! son variantes, porque cumplen las características dadas anteriormente para considerarlas de ese modo, y además son sinónimas.

En definitiva, definimos y analizamos la variación como fenómeno fraseológico no fundamental que puede aparecer en las UFs en modo de variantes o modificaciones.

\section{Referencias bibliográficas}

Alvarado Ortega, M. B. (2006): “¿Son las fórmulas rutinarias enunciados independientes?". En Face, T. L. y C. A. Klee (eds.): Selected Proceedings of the 8th Hispanic Linguistics Symposium. Somerville, Cascadilla Proceedings Project, págs. 214-220.

Alvarado Ortega, M. B. (2007): "iQué bonito! y sus relaciones con la ironía”. En Interlingüística, 17, págs. 114-119.

Alvarado Ortega, M. B. (2008): Las fórmulas rutinarias en el español actual. (Tesis doctoral inédita).

Alvarado Ortega, M. B. y L. Ruiz Gurillo (2006): "Unidades de la conversación y fraseología: acerca de la autonomía de las fórmulas rutinarias". En Actas del I Congreso Internacional sobre Fraseologia y Paremiología. Santiago de Compostela.

Briz, A. y Grupo Val.Es.Co. (2002): Corpus de conversaciones coloquiales. Madrid, Arco Libros.

Burger, H. (2000): "El concepto de variación en fraseología". En A. Pamies y J. D. Luque (eds.): Trabajos de lexicografia y fraseología contrastiva. Granada, Método ediciones, págs. 105-117.

Carneado, Z. y A. M. Tristá (1983): Estudios de fraseología. La Habana, Academia de Ciencias de Cuba.

Casares, J. ([1950] 1969): Introducción a la lexicografía moderna. Madrid, Revista de Filología Española, Anejo LII.

Corpas, G. (1996): Manual de fraseología española. Madrid, Gredos.

Coulmas, F. (ed.) (1981): Conversational Routine. Explorations in Standardized Communication Situations and Prepatterned Speech. Vol. II, La Haya, Mouton.

García-Page, M. (1996): "Sobre las variantes fraseológicas en español". En Revista canadiense de estudios hispánicos, vol. XX, 3, págs. 477-489.

García-Page, M. (1999): "Variantes morfológicas y unidades fraseológicas". En Paremia, 8. Madrid, págs. $225-230$ 
Hernando L. A. (1990): "Sobre las unidades fraseológicas en español". En Actas de la Sociedad Española de Lingüistica. XX Aniversario, Vol. 1, págs. 538-546.

Koike, K. (2001): "Variación fraseológica del español". En Varilex, 9. En línea http://lingua.cc.sophia. ac.jp/varilex/index.php

Montoro del Arco, E. T. (2006): Teoría fraseológica de las locuciones particulares. Las locuciones prepositivas, conjuntivas y marcadoras en español. Frankfurt am Main, Peter Lang.

Moon, R. (1998): Fixed expressions and idioms in English. Nueva York, Oxford University Press.

Ortega Ojeda, G. y M. I. González Aguilar (2005): "En torno a la variación de las unidades fraseológicas". En R. Almela; E. Ramón Trives y G. Wotjak (eds.): Fraseología Contrastiva con ejemplos tomados del alemán, español, francés e italiano. Murcia, Univ. Murcia Servicio de publicaciones, págs. 91-109.

Ruiz Gurillo, L. (1996): “Sobre la fraseología coloquial: corpus e investigación”. En Actas del I Congreso Internacional de AESLA (Granada, septiembre de 1992), págs. 493-498.

Ruiz Gurillo, L. (1997): Aspectos de fraseología teórica española. Valencia, Universidad, Anejo XXIV de Cuadernos de Filología.

Ruiz Gurillo, L. (1998): La fraseologia del español coloquial. Barcelona, Ariel.

Ruiz Gurillo, L. (2001): Las locuciones en español actual. Madrid, Arco Libros.

Ruiz Gurillo, L. (2002a): "La neología nominal sintagmática en el español técnico". En A. Bernabé; J.A. Berenguer; M. Cantanero y J. C. Torres (eds.): Presente y futuro de la Lingüística en España: La sociedad de lingüística 30 años después, II, págs. 449-457.

Ruiz Gurillo, L. (2002b): "Compuestos, colocaciones, locuciones: intento de delimitación". En A. Veiga; M. González y M. Souto (eds.): Lenguas e Lingüística: Léxico y Gramática, 3. Lugo, Tris tram, págs. 325-339.

Zamora, P. (1998): "Las frases idiomáticas pragmáticas italianas: los marcos de situación y sus equivalencias en español". En Estudios románicos. 10, págs. 107-125.

Zamora, P. (1999): "Otro intento de clasificación y delimitación de unidades fraseológicas italianas". En Homenaje al profesor Trigueros Cano. II, Murcia, págs. 733-750.

Zuluaga, A. (1980): Introducción al estudio de las expresiones fijas. Tübingen, Max Hueber, Verlag. (Tesis doctoral inédita).

Zuluaga, A. (1992): "Spanisch: Phraseologie". En Holtus, G; M. Metzeltin y CH. Schmitt (eds.): Lexikon der Romanistischen Linguistik. Tübingen, Max Niemeyer, Verlag, págs. 125-131. 